\title{
One-shot diagnosis: EBUS-TBNA as a single procedure for thyroid, pulmonary and lymph nodal lesions
}

\author{
Niccolò Filippi', Elena Prisciandaro', Juliana Guarize', Stefano Maria Donghi', Giulia Sedda', \\ Lorenzo Spaggiari ${ }^{1,2}$
}

'Department of Thoracic Surgery, IEO, European Institute of Oncology IRCCS, Milan, Italy

${ }^{2}$ Department of Oncology and Hemato-oncology, University of Milan, Milan, Italy

Endobronchial ultrasound-guided transbronchial needle aspiration (EBUS-TBNA) has proved to be the best approach to mediastinal investigation [1], due to its many advantages and low complications rate. It is a minimally invasive procedure, well tolerated by patients, which provides cytological specimens of mediastinal lymph nodes and other central thoracic masses. However, there are few references in literature reporting the use of EBUS-TBNA as a multisampling procedure for the differential diagnosis of thoracic malignancies.

A 56-year-old Caucasian, non-smoker woman with a history of colorectal cancer (CRC) underwent a thoracic computed tomography (CT) showing a 48-mm mass in the right upper pulmonary lobe, an enlargement of the left suprarenal gland and an alteration of the left thyroid lobe, associated with left lateral cervical and mediastinal lymphadenopathies. ${ }^{18} \mathrm{~F}$-fluorodeoxyglucose positron-emission tomography $\left({ }^{18} \mathrm{~F}\right.$-FDG PET) disclosed an intense uptake in all the aforementioned sites (Figure 1). Flexible bronchoscopy revealed a vegetative lesion in the apical segmental bronchus of the right upper lobe; therefore, an endobronchial biopsy was carried out. EBUS-TBNA was performed on the pulmonary lesion, thyroid and right paratracheal lymph node. There were no complications during or after the procedure and, after careful observation, the patient was discharged. Pathological analysis and immunohistochemistry findings were compatible with colorectal adenocarcinoma. After multidisciplinary discussion of the case, the patient started further cycles of chemotherapy.

In patients with a history of previous malignancies, the management of lung nodules or masses associated with systemic lesions represents a challenging issue. In order to distinguish between recurrence of past tumor, new primary cancer or two coexisting neoplasms, pathological sampling of all suspicious sites is usually required. Subjecting the patient to multiple and possibly invasive procedures might result in late diagnosis and late treatment. In the presented case, EBUS-TBNA under conscious sedation was the chosen approach to simultaneous investigation of thyroid, pulmonary and mediastinal lesions in a patient with a history of CRC. Differential diagnosis included: recurrent metastatic CRC, stage IV [2] lung cancer and locally advanced N2 lung cancer associated with colorectal metastases to the thyroid and suprarenal gland. Pathological analysis disclosed poorly differentiated colorectal adenocarcinoma. An ultrasound-guided fine needle aspirate (US-FNA) of the

A

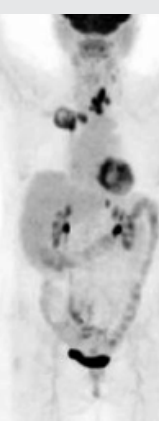

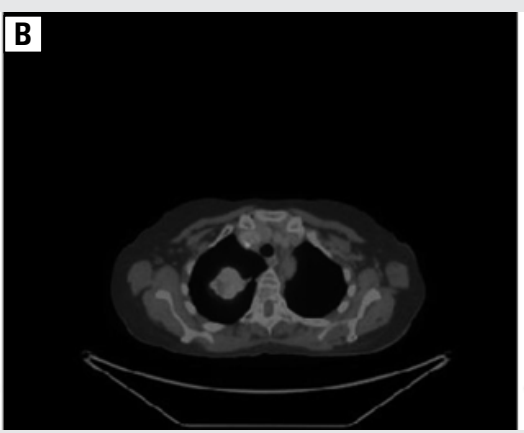

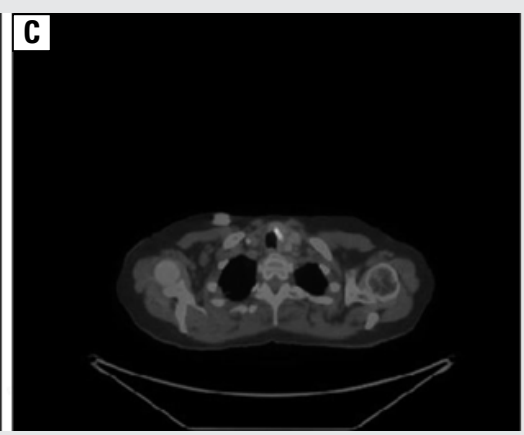

Figure 1. PET findings: A. Coronal view showing multiple sites of increased ${ }^{18} \mathrm{~F}-\mathrm{FDG}$ uptake; B. Axial view of the thorax showing the pulmonary mass in the right upper lobe and the right paratracheal lymph node; C. Axial view of the thorax showing the thyroid lesion

Address for correspondence: Niccolò Filippi, Department of Thoracic Surgery IE0, European Institute of Oncology, Via Ripamonti, 435, 20141 Milan, Italy; e-mail: niccolo.filippi@ieo.it

DOI: 10.5603/ARM.2019.0027

Received: 11.04.2019

Copyright (c) 2019 PTChP

ISSN 2451-4934

Conflict of interest: none declared 
thyroid lesion might have been carried out as well, being a less invasive procedure and not requiring sedation [3]. On the other hand, pathological investigation on the right lung and mediastinal lymph nodes would have been delayed. For the same reason, needle or surgical biopsies of the lung, lateral cervical nodes and suprarenal gland biopsies were not indicated, being unable to provide samples from multiple anatomical sites. Moreover, a surgical approach relates to higher risks of post-operative complications.

Apart from being recommended as the first choice procedure for mediastinal staging in lung cancer, EBUS-TBNA should also be considered in the differential diagnosis of other thoracic malignancies. In the presented case, it allowed to target simultaneously both the thyroid gland and the thoracic lesions. EBUS-TBNA has proved a reliable, safe and time-saving approach to this particular issue, providing a one-shot comprehensive diagnostic evaluation and avoiding further and more invasive investigation.

\section{References:}

1. Guarize J, Casiraghi M, Donghi S, et al. Endobronchial ultrasound transbronchial needle aspiration in thoracic diseases: much more than mediastinal staging. Can Respir J. 2018; 2018: 4269798, doi: 10.1155/2018/4269798, indexed in Pubmed: 29686741.

2. Detterbeck FC, Boffa DJ, Kim AW, et al. The eighth edition lung cancer stage classification. Chest. 2017; 151(1): 193-203, doi: 10.1016/j. chest.2016.10.010, indexed in Pubmed: 27780786.

3. Casal RF, Phan MN, Keshava K, et al. The use of endobronchial ultrasound-guided transbronchial needle aspiration in the diagnosis of thyroid lesions. BMC Endocr Disord. 2014; 14: 88, doi: 10.1186/1472-6823-14-88, indexed in Pubmed: 25416021. 